\title{
TABLE DES MATIÈRES
}

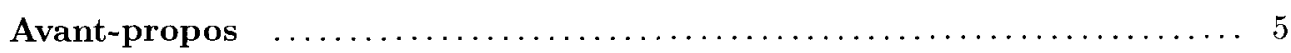

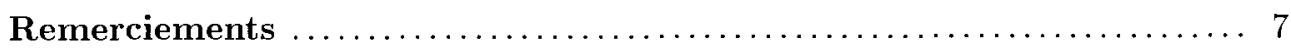

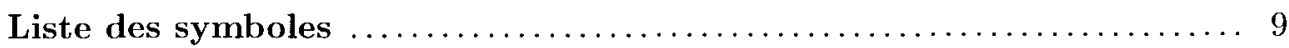

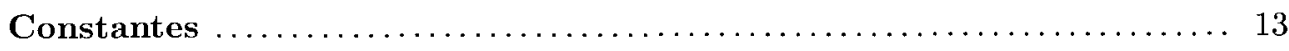

Chapitre 1. Le milieu plasma :

définition et principales grandeurs caractéristiques $\ldots \ldots \ldots \ldots \ldots$

1.1. Définition et nature essentielle du plasma $\ldots \ldots \ldots \ldots \ldots \ldots \ldots \ldots \ldots$

1.1.1. Un plasma est un milieu à comportement collectif $\ldots \ldots \ldots \ldots \ldots 15$

1.1.2. Un plasma est un milieu macroscopiquement neutre $\ldots \ldots \ldots \ldots .16$

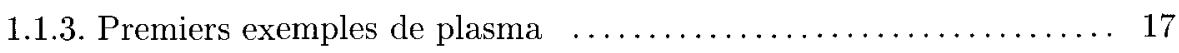

1.2. Domaines d'étude et d'applications (exemples) $\ldots \ldots \ldots \ldots \ldots \ldots \ldots 19$

1.2.1. Fusion thermonucléaire contrôlée $\ldots \ldots \ldots \ldots \ldots \ldots \ldots \ldots \ldots \ldots$

1.2.2. Astrophysique et physique de l'environnement spatial $\ldots \ldots \ldots \ldots 21$

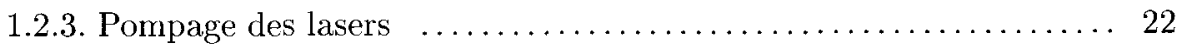

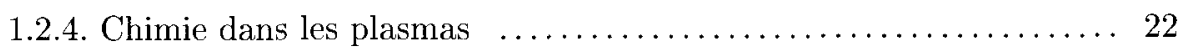

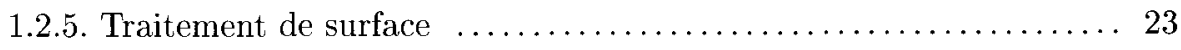

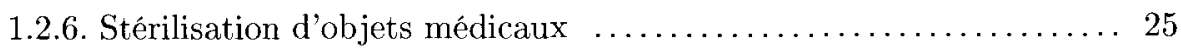

1.2.7. Analyse élémentaire (chimie analytique) $\ldots \ldots \ldots \ldots \ldots \ldots \ldots 26$

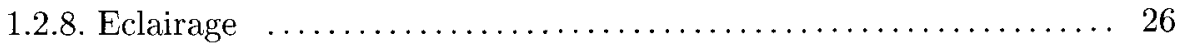

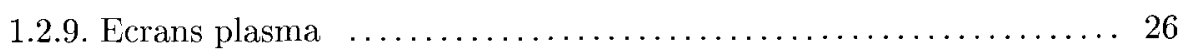

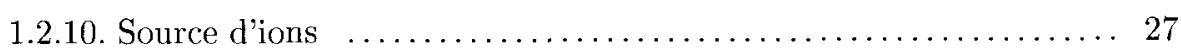

1.3. Différents types de décharge en laboratoire $\ldots \ldots \ldots \ldots \ldots \ldots \ldots \ldots 27$ 
1.3.1. La décharge en courant continu ou alternatif de basse fréquence $\ldots 27$

1.3.2. La décharge de haute fréquence $(\mathrm{HF}) \ldots \ldots \ldots \ldots \ldots \ldots \ldots \ldots \ldots$

1.3.3. La décharge par rayonnement laser $\ldots \ldots \ldots \ldots \ldots \ldots \ldots \ldots \ldots \ldots$

1.4. Densité électronique et température d'un plasma $\ldots \ldots \ldots \ldots \ldots \ldots .28$

1.4.1. Domaine des valeurs de densité électronique des plasmas $\ldots \ldots \ldots 28$

1.4.2. Concept d'équilibre thermodynamique (ET)

et définition de la "température" d'un plasma $\ldots \ldots \ldots \ldots \ldots \ldots 29$

1.4.3. Divers niveaux d'écart par rapport

à l'équilibre thermodynamique complet $\ldots \ldots \ldots \ldots \ldots \ldots \ldots . \ldots \ldots 2$

1.5. Fréquence propre d'oscillation des électrons d'un plasma $\ldots \ldots \ldots \ldots .34$

1.5.1. Origine et description du phénomène $\ldots \ldots \ldots \ldots \ldots \ldots \ldots \ldots . \ldots \ldots$

1.5.2. Calcul de la fréquence propre des électrons du plasma . . . . . . 35

1.6. Longueur de DeByE : effet d'écran dans les plasmas $\ldots \ldots \ldots \ldots \ldots \ldots 38$

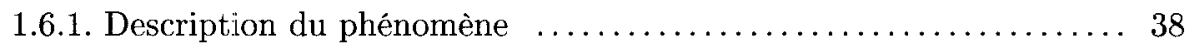

1.6.2. Calcul du potentiel d'un ion dans un plasma à deux températures : définition de la longueur de DEBYE $\ldots \ldots \ldots \ldots 38$

1.7. Phénomènes de collision dans les plasmas $\ldots \ldots \ldots \ldots \ldots \ldots \ldots \ldots \ldots$

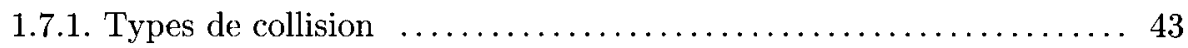

1.7.2. Echange de quantité de mouvement et transfert d'énergie lors d'une collision entre deux particules $\ldots \ldots \ldots \ldots \ldots \ldots \ldots .46$

1.7.3. Section efficace microscopique différentielle $\ldots \ldots \ldots \ldots \ldots \ldots \ldots$

1.7.4. Section efficace microscopique intégrée (totale) $\ldots \ldots \ldots \ldots \ldots 57$

1.7.5. Section efficace macroscopique totale $\ldots \ldots \ldots \ldots \ldots \ldots \ldots \ldots$

1.7.6. Expression de la température d'un plasma en électron-volt ..... 62

1.7.7. Fréquence de collision et libre parcours probable

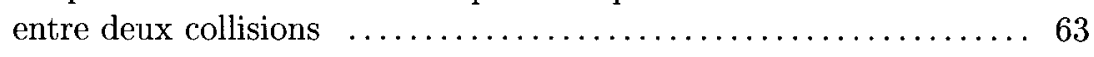

1.7.8. Fréquence moyenne de collision et libre parcours moyen ....... 64

1.7.9. Exemples de sections efficaces collisionnelles $\ldots \ldots \ldots \ldots \ldots \ldots 66$

1.8. Mécanismes de perte et de création des particules chargées d'un plasma et leur équation de conservation $\ldots \ldots \ldots \ldots \ldots \ldots \ldots \ldots$

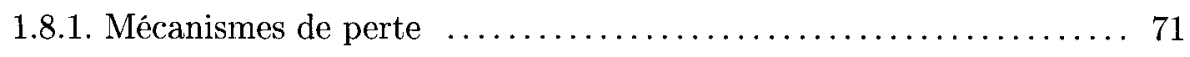

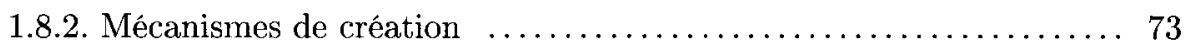

1.8.3. Equation de conservation des particules chargées $\ldots \ldots \ldots \ldots \ldots 74$ 
Chapitre 2. Mouvement individuel d'une particule chargée dans des champs électrique et magnétique

2.1. Equation générale du mouvement d'une particule chargée dans des champs $\boldsymbol{E}$ et $\boldsymbol{B}$ et propriétés de cette équation $\ldots \ldots \ldots \ldots 77$

2.1.1. Equation du mouvement $\ldots \ldots \ldots \ldots \ldots \ldots \ldots \ldots \ldots \ldots \ldots \ldots \ldots \ldots$

2.1.2. Equation des forces vives $\ldots \ldots \ldots \ldots \ldots \ldots \ldots \ldots \ldots \ldots \ldots \ldots \ldots \ldots \ldots \ldots$

2.2. Analyse de cas particuliers de $\boldsymbol{E}$ et $\boldsymbol{B} \ldots \ldots \ldots \ldots \ldots \ldots \ldots \ldots$

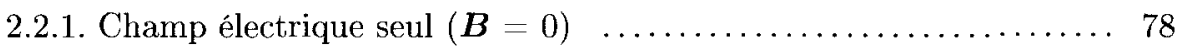

2.2.2. Champ magnétique constant et uniforme $\ldots \ldots \ldots \ldots \ldots \ldots \ldots$

2.2.3. Champ magnétique non uniforme ou variable dans le temps .... 106

Chapitre 3. Description hydrodynamique d'un plasma $\ldots \ldots \ldots \ldots \ldots \ldots \ldots . \ldots$

3.1. Considérations élémentaires sur l'équation de Boltzmann ........ 127

3.1.1. Présentation sommaire de l'équation de Boltzmann $\ldots \ldots \ldots \ldots 127$

3.1.2. Approximation du terme de collisions élastiques de Boltzmann : relaxation de la fonction de distribution vers un état isotrope . . 129

3.1.3. Deux méthodes classiques de recherche de solution de l'équation de Boltzmann $\ldots \ldots \ldots \ldots \ldots \ldots \ldots \ldots \ldots \ldots \ldots \ldots \ldots \ldots$

3.2. Fonctions de distribution des vitesses et notions de corrélation $\ldots \ldots, 132$

3.2.1. Densité de probabilité de présence $\ldots \ldots \ldots \ldots \ldots \ldots \ldots \ldots \ldots \ldots \ldots$

3.2.2. Fonction de distribution simple (cas de particules corrélées) . ... 133

3.2.3. Fonction de distribution simple (cas de particules non corrélées) 134

3.2.4. Fonction de distribution double (cas de particules corrélées) . ... 134

3.2.5. Fonction de distribution double (cas de particules non corrélées) 135

3.2.6. Fonction de distribution à $N$-tuples $\ldots \ldots \ldots \ldots \ldots \ldots \ldots \ldots \ldots$

3.3. Fonctions de distribution et grandeurs hydrodynamiques $\ldots \ldots \ldots \ldots .136$

3.4. Conductivité cinétique et hydrodynamique des électrons d'un plasma en présence d'un champ électromagnétique $\mathrm{HF} \ldots \ldots \ldots \ldots \ldots \ldots \ldots 139$

3.4.1. Forme cinétique de la conductivité électrique due aux électrons en champ $\mathrm{HF} \ldots \ldots \ldots \ldots \ldots \ldots \ldots \ldots \ldots, 139$

3.4.2. Forme hydrodynamique de la conductivité électrique due aux électrons en champ $\mathrm{HF} \ldots \ldots \ldots \ldots \ldots \ldots \ldots \ldots \ldots \ldots \ldots$

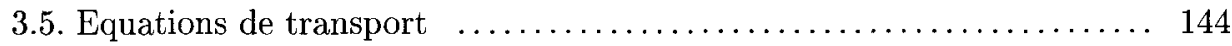

3.5.1. Equation de continuité ( $1^{\mathrm{er}}$ moment hydrodynamique :

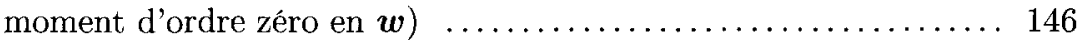


3.5.2. Equation de transport de quantité de mouvement $\left(2^{\mathrm{e}}\right.$ moment :

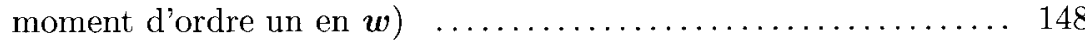

3.5.3. Equations du moment d'ordre 2 en $\boldsymbol{w} \ldots \ldots \ldots \ldots \ldots \ldots \ldots \ldots$

3.5.4. Equation des moments d'ordre supérieur $\ldots \ldots \ldots \ldots \ldots \ldots \ldots$

3.6. Fermeture des équations de transport $\ldots \ldots \ldots \ldots \ldots \ldots \ldots \ldots \ldots \ldots \ldots$

3.7. Modèle du plasma d'électrons de Lorentz $\ldots \ldots \ldots \ldots \ldots \ldots \ldots \ldots . . \ldots 1$

3.8. Mobilité et diffusion de particules chargées $\ldots \ldots \ldots \ldots \ldots \ldots \ldots \ldots . \ldots 3$

3.8.1. Les concepts de diffusion et de mobilité $\ldots \ldots \ldots \ldots \ldots \ldots \ldots . \ldots 16$

3.8.2. Solution de l'équation de LANGEviN avec dérivée particulaire nulle $(\mathrm{d} v / \mathrm{d} t=0) \quad \ldots \ldots \ldots \ldots \ldots \ldots \ldots \ldots \ldots \ldots$

3.9. Modes propres de diffusion et distribution spatiale de la densité des particules chargées $\ldots \ldots \ldots \ldots \ldots \ldots \ldots \ldots \ldots \ldots . \ldots \ldots$

3.9.1. Notions de modes propres de diffusion : étude d'une post-décharge temporelle

3.9.2. Distribution spatiale de la densité des particules chargées en régime stationnaire de diffusion

3.10. Diffusion en régime ambipolaire

3.10.1. Hypothèses nécessaires à une description analytique complète du régime de diffusion ambipolaire

3.10.2. Equations régissant la diffusion ambipolaire et le régime de transition de la diffusion libre vers la diffusion ambipolaire $\quad . \quad 179$

3.10.3. Valeur de l'intensité du champ électrique de charge d'espace $\ldots 181$

3.10.4. Expression de la densité des charges $\rho_{0}$ sur l'axe: limite de validité du calcul analytique

3.10.5. Conditions à remplir pour qu'une décharge en mode de diffusion soit en régime ambipolaire $\ldots \ldots \ldots \ldots \ldots 184$

3.11. Diffusion ambipolaire en champ magnétique statique $\ldots \ldots \ldots \ldots \ldots 187$

3.12. Régime de diffusion ou de chute libre ? .................... 188

3.13. Température électronique d'une longue colonne de plasma régi par la diffusion ambipolaire : loi d'échelle $T_{e}(p R) \ldots \ldots \ldots \ldots 190$

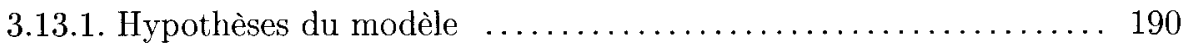

3.13.2. Dérivation de la relation $T_{e}(p R) \quad \ldots \ldots \ldots \ldots \ldots \ldots \ldots \ldots \ldots \ldots \ldots$

3.14. Formation et nature des gaines à l'interface plasma-paroi : flux aux parois et critère de Вонм $\ldots \ldots \ldots \ldots \ldots \ldots \ldots \ldots \ldots \ldots \ldots \ldots$

3.14.1. Cas d'un potentiel de paroi positif par rapport au potentiel du plasma : gaine électronique 
3.14.2. Cas d'un potentiel de paroi négatif par rapport au potentiel de plasma : gaine ionique ........ 199

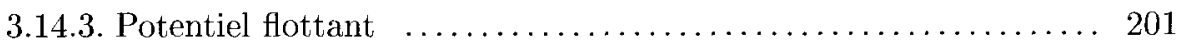

Chapitre 4. Introduction à la physique des décharges $\mathrm{HF} \quad \ldots \ldots \ldots \ldots \ldots 203$

4.1. Préambule ............................................. 203

4.2. Transfert de puissance du champ électrique à la décharge $\ldots \ldots \ldots \ldots 205$

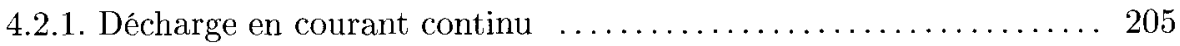

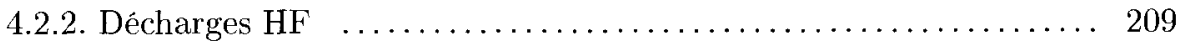

4.2.3. Décharges HF en présence d'un champ magnétique statique $\ldots . .211$

4.2.4. Evolution de la valeur de $\theta$ en fonction de $\bar{n}_{e}$ dans diverses conditions de plasma $\ldots \ldots \ldots \ldots \ldots \ldots \ldots \ldots 217$

4.3. Influence de la fréquence du champ HF sur quelques propriétés du plasma et sur certains procédés $\ldots \ldots \ldots \ldots 219$

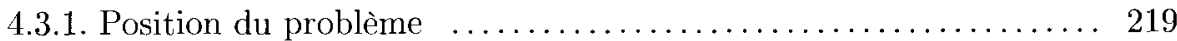

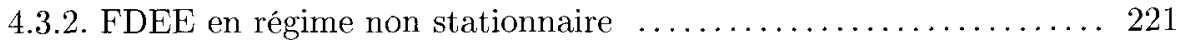

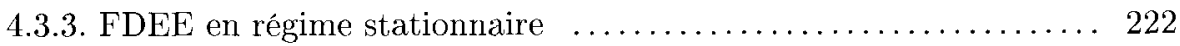

4.3.4. Trois cas limites de l'influence de $\omega$ sur la FDEE stationnaire . . 223

4.3.5. Influence de $\omega$ sur la valeur de la puissance $\theta \quad \ldots \ldots \ldots \ldots \ldots 226$

4.3.6. Densité d'espèces produites par seconde à densité de puissance absorbée constante : efficacité énergétique $\ldots \ldots \ldots \ldots \ldots \ldots, 227$

4.3.7. Résultats expérimentaux et modélisation $\ldots \ldots \ldots \ldots \ldots \ldots \ldots 227$

4.3.8. Conclusion sommaire à l'étude des propriétés des plasmas HF

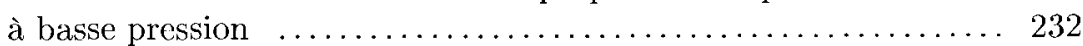

4.4. Les plasmas $H F$ à haute pression $\ldots \ldots \ldots \ldots \ldots \ldots \ldots \ldots \ldots \ldots . \ldots \ldots 232$

4.4.1. Observation expérimentale des phénomènes de contraction et de filamentation à la pression atmosphérique $\ldots \ldots \ldots \ldots \ldots .233$

4.4.2. Hypothèses d'étude du phénomène de contraction

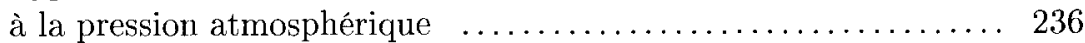

4.4.3. Validation par un modèle auto-cohérent des hypothèses émises sur la contraction à la pression atmosphérique $\ldots \ldots \ldots \ldots \ldots .240$

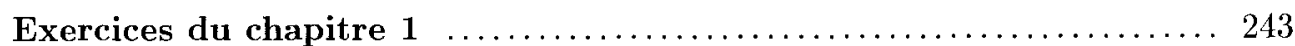

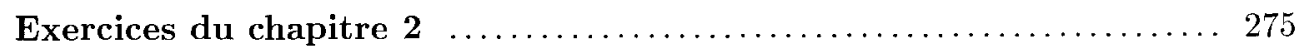

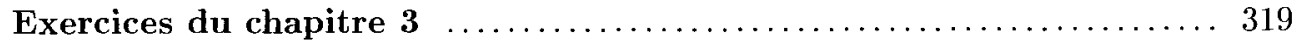


Annexes 363

Annexe I. Rappels sur la fonction de distribution des vitesses de Maxwell-Boltzmann (M-B)

Annexe II. Expression complète de l'équation de SAHA 367

Annexe III. Equilibre thermodynamique local partiel 368

Annexe IV. Représentation des collisions binaires dans les repères du centre de masse et du laboratoire

Annexe V. Interactions collisionnelles de nature coulombienne.

Limitation de leur portée (logarithme coulombien)

Annexe VI. Ionisation par étape

Annexe VII. Notions de tenseur

Annexe VIII. Opérations sur les tenseurs

Annexe IX. Orientation de $\boldsymbol{w}_{2 \perp}$ dans le trièdre de référence $\left(\boldsymbol{E}_{0 \perp} \wedge \boldsymbol{B}, \boldsymbol{E}_{0 \perp}, \boldsymbol{B}\right)$

Annexe X. Force agissant sur une particule chargée dans la direction d'un champ $\boldsymbol{B}$ faiblement non uniforme axialement : variante de $(2.172)$

Annexe XI. Le moment magnétique, un invariant dans l'approximation du centre de guidage

Annexe XII. Vitesse de dérive $\boldsymbol{w}_{\mathrm{D}}$ d'une particule chargée soumise à une force quelconque $\boldsymbol{F}_{\mathrm{D}}$ dans un champ $\boldsymbol{B}$ :

la dérive magnétique 396

Annexe XIII. Vitesse de dérive magnétique $\boldsymbol{w}_{\mathrm{DM}}$ dans le repère de FreneT associé aux lignes de force d'un champ magnétique présentant une courbure

Annexe XIV. Harmoniques sphériques 400

Annexe XV. Expression des termes $\underline{\boldsymbol{M}}$ et $\underline{\mathcal{R}}_{\alpha}$ de l'équation de transport de la pression cinétique (3.151) 
Annexe XVI. Fermeture de l'équation hydrodynamique de transport de pression cinétique dans le cas d'une compression adiabatique 402

Annexe XVII. Compléments de calcul pour l'expression de $T_{e}(p R)(\S 3.13)$. 403 Annexe XVIII. Plasmas d'onde de surface (POS) $\ldots \ldots \ldots \ldots \ldots \ldots \ldots \ldots$

Annexe XIX. Intégrales utiles et expressions des principaux opérateurs différentiels 408

Bibliographie 413

Références 415

Index 417 